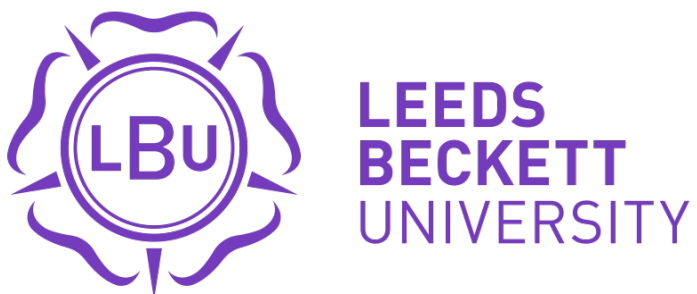

Citation:

Kor, A and Pattinson, C and Yanovsky, M and Kharchenko, V (2017) SMART-ITEM: IoT-Enabled Smart Living. IEEE. DOI: https://doi.org/10.1109/FTC.2016.7821687

Link to Leeds Beckett Repository record:

https://eprints.leedsbeckett.ac.uk/id/eprint/4104/

Document Version:

Article (Accepted Version)

\section{Conference proceeding}

The aim of the Leeds Beckett Repository is to provide open access to our research, as required by funder policies and permitted by publishers and copyright law.

The Leeds Beckett repository holds a wide range of publications, each of which has been checked for copyright and the relevant embargo period has been applied by the Research Services team.

We operate on a standard take-down policy. If you are the author or publisher of an output and you would like it removed from the repository, please contact us and we will investigate on a case-by-case basis.

Each thesis in the repository has been cleared where necessary by the author for third party copyright. If you would like a thesis to be removed from the repository or believe there is an issue with copyright, please contact us on openaccess@leedsbeckett.ac.uk and we will investigate on a case-by-case basis. 


\title{
SMART-ITEM: IoT-Enabled Smart Living
}

\author{
Ah-Lian Kor \\ School of Computing, Creative Technologies, and Engineering \\ Leeds Beckett University \\ Leeds, UK \\ A.Kor@leedsbeckett.ac.uk \\ Colin Pattinson \\ School of Computing, Creative Technologies, and Engineering \\ Leeds Beckett University \\ Leeds, UK \\ Max Yanovsky \\ Department of Computer Systems and Networks, \\ National Aerospace University "KhAl", \\ Kharkiv, Ukraine \\ Vyacheslav Kharchenko \\ Department of Computer Systems and Networks, \\ National Aerospace University "KhAl”, \\ Kharkiv, Ukraine
}

\begin{abstract}
The main goal of this proposed project is to harness the emerging loT technology to empower elderly population to selfmanage their own health, stay active, healthy, and independent as long as possible within a smart and secured living environment. An integrated open-sourced loT ecosystem will be developed. It will encompass the entire data lifecycle which involves the following processes: data acquisition, data transportation; data integration, processing, manipulation and computation; visualisation; data intelligence and exploitation; data sharing; data storage. This innovative cloud-based loT ecosystem will provide a one-stop shop for integrated smart loTenabled services to support older people (greater or equal to 65 years old) who live alone at home (or care homes). Another innovation of this system is the design and implementation of an integrated loT gateway for wellebing wearable and home automation system sensors with varying communication protocols. The SMART-ITEM system and services will appropriately address the following (i) smart health and care; (ii) smart quality of life; (iii) SMART-ITEM social community. The development of the system will be based on the User Centred Design methodology so as to ensure active user engagement throughout the entire project lifecycle and necessary standards as well as compliances will be adhered to (e.g. security, trust and privacy) in order to enhance user acceptance.
\end{abstract}

Keywords-IoT, Smart Home, Smart Health, Architecture, Agile methodology, Smart Ssystem and Services, Independent Living

\section{INTRODUCTION}

By 2020, a quarter of Europeans will be over 60 years of age and this will impact on our health care, economy, and social security systems ${ }^{1}$. According to EC, Europe is already spending nearly $10 \%$ GDP on healthcare due to EU ageing population ${ }^{2}$. Consequently, the EU Health $2020^{3}$ aims to: "significantly improve the health and well-being of populations, reduce health inequalities, strengthen public health and ensure people-centred health systems that are universal, equitable, sustainable and of high quality". In order to support Action Plan for Healthy Ageing ${ }^{4}$, this proposal aims to provide an inclusive, integrated and usercentric loT-ecosystem and services to promote smart health care, smart quality of life, and smart social inclusion.

${ }^{1}$ https://ec.europa.eu/research/social-sciences/pdf/policy_reviews/kina26426enc.pdf

${ }^{2}$ http://ec.europa.eu/research/participants/portal/doc/call/h2020/common/1587763-08. health_wp2014-2015_en.pdf

${ }^{3}$ http://www.euro.who.int/en/health-topics/health-policy/health-2020-the-european-policy-for-health-and-well-being/about-health-2020

${ }^{4}$ http://www.euro.who.int/_data/assets/pdf_file/0008/175544/RC62wd10Rev1-Eng.pdf 


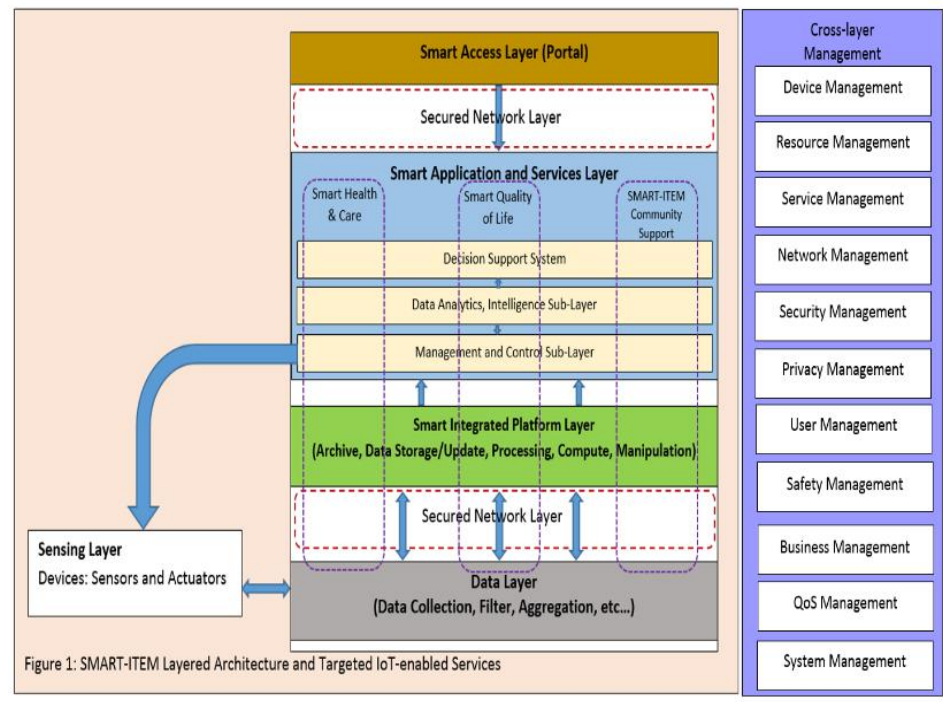

\section{A. Project Objectives}

The following list of concrete objectives will help achieve the afore-mentioned goals of SMART-ITEM System and Services:

Objective 1: Provide a user-centred service where users are co-creators of good health In order to ensure user-centeredness and co-creation, users are extensively involved throughout SMART-ITEM iterative development lifecycle (i.e. requirements phase, development phase, evaluation phase and integration). Users will be empowered to make health related decisions (with or without clinicians) and responsibly act on information provided in their respective secured e-health passports. Access to SMARTITEM services is facilitated via multi-modal (and lingual) facility and devices (PDAs, mobile phones, tablets, laptops, desktops, etc...);

Objective 2: Provide an integrated one-stop shop portal for loT-enabled services for smart living within a secured living environment The rationale for such a portal is to: co-ordinate the multiple services provision; afford a business model which offers users easy access to multiple services in one 'location';

Objective 3: Provide real-time user health information and analysis User's self-monitoring health data (e.g. through multi-sensor physiological monitoring - heart rate, respiration, temperature, oxygenation, etc...) will be securely transmitted (via wireless technologies), analysed in real-time (by an intelligent data system), and fed back via the e-health passport application. Permission will be granted by the user to a third party so that they could view the e-health passport information (e.g. clinicians, family members, carers, etc...);

Objective 4: Provide synchronous and asynchronous user activity information and analysis Wearable activity tracker could be employed to track physical and health activities (e.g. steps taken, stairs climbed, sleep hours and quality logged, and distance travel, etc...);

Objective 5: Provide an loT-based smart, safe, and secured living environment (i.e. smart homes) This will help: manage temperature, air quality, etc...; detect falls, intrusion, gas leaks, etc...

Objective 6: Build an online Community for users The community will comprise smart living experts and users (that are stratified based on needs, demographics and languages). The primary communication channel will be via social media. It aims to: facilitate knowledge transfer (from experts to novices); knowledge/experience sharing (amongst users with similar needs); facilitate social interaction, engagement, and integration; provide emotional support;

Objective 7: Bring about benefits to users and stakeholders A range of impact analyses will be conducted to determine the benefits of SMART-ITEM (e.g. cost effective analysis, economic impact analyses, user satisfaction, environmental impact analysis, health economics analysis, financial performance analysis of health economy, etc...).

In summary, in this project, we shall seize a list of opportunities: (i) empower elderly population to manage their own health and stay active, healthy and independent as long as possible; (ii) development of loT-enabled systems which are people-centred and home based integrated care; (iii) support an economy which adapts to new categories of clients and their needs (note: with ICT-driven innovation we can tap into the growing Silver economy of 85 million consumers over 65 and $€ 3000$ billion). 


\section{RELATED WORK}

IBM's vision of s smarter home enabled by cloud technology 5 has the following characteristics: Instrumented - the ability to sense and monitor changing conditions; Interconnected - the ability to communicate and interact, with people, systems and other objects; Intelligent - the ability to make decisions based on data, leading to better outcomes. According to IBM, the categories of smarter home services are as follows: Entertainment and Convenience - the internet-enabled television will processed to personalised entertainment content via a portal; Energy Management - management of home electrical appliances to deliver automatic savings to home owners (e.g. automatically synchronised lighting, climate control sensors, etc...); Safety and Security - as an example, deployment of centralised alarm services using sensors and cameras and instant notification of relevant parties could enhance home security; Health and Wellness - smarter home scales and sensors monitor fitness, well-being and consistent activities. These home devices could collect evaluative information about current health condition for disease prevention and overall wellness. In SMART-ITEM, we shall categories smart home services into three categories which are integrated into a onestop-web-portal for ease of use: Smart Health and Care; Smart Quality of Life; SMART-ITEM and Community of Support.

\section{Smart Health and Care}

The domain of e-health is extended with wearables or mobile loT devices for home-based or on-the-go (mobile) monitoring of vital data ${ }^{6}$. Some examples of these devices are: wirelessly connected glucometers, heart rate and blood pressure monitors, scales, oximeter, etc...Tracking health and wellbeing information is vital for the elderly population. However, current ehealth systems require users to manually input their vital data through smart phones or tablets (e.g. Vitrucare System ${ }^{7}$ by Dynamic Health Systems, Wellness Layers Digital Therapeutic Platform ${ }^{8}$, Honeywell Lifecare Solutions ${ }^{9}$ (e.g. Honeywell HomMed), Phillips eTraC program and eCare Companion ${ }^{10}$, etc...). Garmin ${ }^{11}$ and Fitbit activity and sleep trackers with their fitness apps afford the following facilities ${ }^{12}$ : progress and performance of all day activity, sleep goals and trends, stay connected with family and friends, provides guidance and tools for nutrition and weight goals, and access through smartphones or desktops. In the same vein, Garmin activity trackers, ${ }^{13}$ smart scales and apps ${ }^{14}$ provides the following functionalities: personalised activity goals, track progress and performance, analysis of sleep trends, wellness and weight management, heart-rate analysis, and smart notifications. A synergy of loT and cloud-based technologies will provide the means for smart devices to automatically transmit, and platforms to analyse as well as store data. This will automatically remove the limitations of manual data collection. In August 2013, Cooking Hacks launched a new version of the first biometric shield for Arduino and Raspberry Pi: the e-Health Sensor Platform V2.0 ${ }^{15}$ which allows users to run biometric and medical applications for body monitoring using 10 different sensors connected to the shield via cables: pulse, oxygen in blood (SPO2), airflow (breathing), body temperature, electrocardiogram (ECG), glucometer, galvanic skin response (GSR - sweating), blood pressure (sphygmomanometer), patient position (accelerometer) and muscle/eletromyography sensor (EMG). Biometric information gathered is then wirelessly transmitted to the cloud using one of the following 6 connectivity options: Wi-Fi, 3G, GPRS, Bluetooth, 802.15.4 and ZigBee depending on the type of application. The cloud will provide the following facilities: permanent storage; processing and analysis platform. Analysed data could be visualised in real time and sent back to a laptop or smartphone (with iPhone or Android OS).

SMART-ITEM e-health and activity differentiation is as follows: (i) low-cost sensing, gateway, and cloud-based platform system (e.g. for SMART-ITEM estimated total cost for weight and SPO2 monitoring is f205.98 - Raspberry Pi 3 Model B (f26 per unit); iHealth PO3 Wireless Pulse Oximeter ( $f 79.99$ per unit); iHealth HS6 Body Analysis Wireless Scale ( $f 99.99$ per unit). On the other

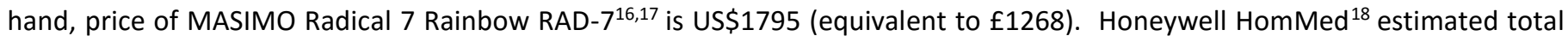
costs is US\$26,350 (equivalent to $\mathrm{f18680)}$ - Genesis DM Pro BP, wireless scale and SPO2 Kit (US\$4500 per unit), Genesis Touch Kit (US\$1000 per unit), Genesis DM peripherals and cables (GPRS - US\$450; Software for LifeStream Base Platform US\$5000; LifeStream Connect US\$10000; Training (Clinical Strategic Planning Meeting Onsite US\$1800; Clinical Strategic Planning Meeting via teleconference US\$600; Clinical Training Initial Day-Onsite US\$1800; Additional Consecutive Day US\$1200); (ii) automated wireless data transfer (from devices to gateway and finally the cloud) to reduce the risk of errors; (iii) allows a single point of access to a range of services and apps; (iv) interoperability with a variety of medical and fitness devices including their natively

${ }^{5}$ http://www.ibm.com/smarterplanet/global/files/uk_uk_en_cloud_a_smarter_home_enabled_by_cloud_computing.pdf

${ }^{6}$ http://www.itu.int/en/ITU-T/academia/kaleidoscope/2014/Pages/SpecialSessionE-health,-IoT-and-Cloud.aspx

${ }^{7}$ http://www.dynamichealthsystems.co.uk/vitrucare-supported-selfcare

8 http://www.wellnesslayers.com/solution-3-2/

${ }^{9}$ https://www.linkedin.com/company/honeywell-hommed

${ }^{10}$ http://www.usa.philips.com/b-dam/b2bhc/master/Products/Category/enterprise-telehealth/etrac/eTrAC.pdf

${ }_{11}^{11}$ http://sites.garmin.com/en-GB/wellness/

${ }^{12}$ http://www.fitbit.com/uk/app

${ }^{13} \mathrm{https} / / /$ buy.garmin.com/en-US/US/wearables/activity-tracking/c10002-atFILTER_USERPROFILE_ACTIVITYTRACKING_01-p1.html

${ }^{14}$ https://buy.garmin.com/en-US/US/prod530464.html

${ }_{15}^{15}$ https://www.cooking-hacks.com/documentation/tutorials/ehealth-biometric-sensor-platform-arduino-raspberry-pi-medical/

${ }^{16} \mathrm{http}: / /$ masimo.co.uk/rainbow/radical7.htm

${ }^{17} \mathrm{http}: / /$ www.ebay.com/itm/MASIMO-Radical-7-Rainbow-RAD-7-Factory-Refurbished-in-the-box-/152023656222

${ }^{18}$ http://www.vnaa.org/Files/CorporatePartnerships/MVPDocuments/Honeywell-2013-pricing.pdf 
available apps; (v) HIPAA compliance ${ }^{19}$;(vi) use cloud-based service-oriented platform that adopts the Service Oriented Architecture (SOA) approach which is a paradigm for organising and utilising distributed capabilities that is under the control of different ownership domains and implemented using various technology stacks ${ }^{20}$. Such architecture is scalable and modular where its services are accessible from external applications via Application Programming Interfaces (APIs).

In summary, SMART-ITEM will be an e-health and wellbeing innovation because it will offer a low-cost solution which harnesses the power of wireless data transfer, synergy of private, public, and hybrid cloud-based computational power, aggregation, and storage, as well as display of analysed active living-related data which is not accessible before. Users' vital and activity data will be collected over time and and analysed using a range of complex algorithms. The analysed data could be sent to caregiver (note: this is an optional functionality) for further analysis and review. This could be exploited to enable preventive care and allow prompt diagnosis of possible complications. SMART-ITEM decision support system will help users make appropriate decisions based on recommendations given by system. SMART-ITEM will support local council's promotion for active ageing for the elderly population (e.g. Age Friendly Leeds ${ }^{21}$, Leeds Older and Active ${ }^{22}$, etc...).

\section{Smart Quality of Life}

The category will encompass the following: smart home automation; safety and security. Smart devices can be used to collect relevant data about the home. As an example, smart thermostats could collect data about occupancy and homeowners' temperature preferences followed by appropriate adjustments. Samsung has launched the Smart Home Ecosystem ${ }^{23}$ which connects home devices (e.g. televisions, home appliances and smartphones) using a single integrated platform. These devices can be controlled by users through an application which connects the devices in a home. Samsung SmartThings Starter Kit ${ }^{24}$ provides everything needed to create a smart home. It is iOS, Android and Windows Phone compatible. SmartThings Starter Kit provides the following functionalities: (i) home monitoring from anywhere; (ii) home control with an app; (iii) home security and protection from damage and danger;(iv) scalability by increasing hundreds of compatible smart devices to enhance home automation. Its components are: hub to connect smart sensors, lights, locks, cameras, etc...; motion sensor to monitor movement in the home; multi-sensor - to monitor whether doors, windows, drawers or garage are open or closed; presence sensor to know when people, pets, and cars arrive or leave home; and a power outlet to control lights, electronics, and small appliances. The Smart Home Cloud API $^{25}$ provides methods to control and monitor Samsung Smart Home devices. Through this Smart Home Control Service, a partner's application can connect with various devices and provide enhanced services to their customer. This service operates through Cloud-to-Cloud integration between the Partner Cloud and Smart Home Cloud. This is facilitated by the provision of several REST API by Samsung for partners so they are able to integrate their system to the Samsung Smart Home Cloud. The body of the REST API uses a standard JSON document (called Smart Home Data) and this means that partner developers will need to understand the JSON document.

Apple Homekit ${ }^{26}$ is Apple's vision for the future smart home with a set of interconnecting apps and devices (via Bluetooth or WiFi technologies) that control lighting, heating and the cycles of your washing machine. HomeKit is a framework ${ }^{27}$ for communicating with and controlling connected accessories in a user's home. Users can configure HomeKit accessories in the home or create actions to control those devices, group actions together and trigger them using an iOS device ${ }^{28}$ or Siri (an Apple personal voice assistant) ${ }^{29}$. Thus, it is a technology platform which cannot be bought because it is aimed at app developers and manufacturers but consumers can only buy HomeKit-compatible gadgets. The focal point of the HomeKit framework is a unifying protocol (i.e. standardisation via a singular SDK) to act as the conduit for devices or gadgets to communicate with iOS ${ }^{30}$, and to make a public API for the configuration and communication with these devices ${ }^{31}$. The Object Orientation (OO) or ontological model has been used to organise the hierarchy of classes and subclasses of objects and thus it is essential for developers to understand the relationships amongst the objects in the model. Finally, Homekit is privacy compliant because Homekit data is always encrypted so that only people in the household the settings of the lights and thermostats. A list of accessories that are Homekit- enabled is found here ${ }^{32}$.

\footnotetext{
${ }^{19} \mathrm{http}: / /$ technologyadvice.com/blog/healthcare/the-importance-of-hipaa-compliance-and-health-app-security/

${ }^{20} \mathrm{http} / / /$ www.adobe.com/enterprise/pdfs/Services_Oriented_Architecture_from_Adobe.pdf

${ }^{21} \mathrm{https}$ ://betterlivesleeds.wordpress.com/age-friendly-leeds/

${ }^{22}$ http://www.altogetherbetter.org.uk/leeds-older-and-active

${ }^{23}$ http://www.computerweekly.com/news/2240212008/Samsung-launches-Smart-Home-ecosystem-to-connect-home-devices

${ }^{24}$ http://www.samsung.com/uk/consumer/smartthings/kit/kit/F-STR-KIT-UK/

${ }^{25} \mathrm{http}: / /$ developer.samsung.com/smart-home

${ }^{26} \mathrm{http} / / / \mathrm{www} . w a r e a b l e . c o m / s m a r t-h o m e / a p p l e-h o m e k i t-e s s e n t i a l-g u i d e-2016$

${ }^{27}$ https://developer.apple.com/homekit/

${ }^{28} \mathrm{http} / / / \mathrm{www}$. apple.com/uk/ios/homekit/

${ }^{29} \mathrm{http://www.apple.com/uk/ios/siri/}$

${ }^{30} \mathrm{http}: / /$ www.programmableweb.com/news/how-to-get-started-apple-homekit/how-to/2014/11/28

${ }^{31}$ https://developer.apple.com/library/ios/documentation/HomeKit/Reference/HomeKit_Framework/

${ }^{32}$ http://www.apple.com/uk/shop/accessories/all-accessories/home-automation?page $=1$
} 
Homebridge $^{33}$ is a lightweight NodeJS server runs on a home network and emulates the iOS HomeKit API. Raspberry PIs running Homebridge can create HomeKit smart home ${ }^{34}$ which controls Samsung SmartThings, Philips Hue lighting and Sonos. RaZberry ${ }^{35}$ uses the Raspberry PI platform with Z-Wave which is the leading wireless communication technology for smart homes. The Razberry platform adds all the components needed to turn a Raspberry PI board into a fully operational and inexpensive Z-Wave gateway. They are: (i) hardware and operating system - Raspberry PI board and Rasperian OS; (ii) Z-wave hardware - RaZberry daughter card which connects to the GPIOs of Raspberry and carry the Sigma Designs Z-Wave transceiver; (iii) Z-wave firmware - runs on the transceiver and is compatible to the original Sigma Designs firmware spec; (iv) Z-wave stack first certified Z-Wave communication stack, handles all Z-Wave network communication, Java script automation engine, built in web server; (v) user interface - web based Z-Way Demo User Interface using the Z-Way JSON API and demonstrating all functions of Z-Way; (vi) user application. Bluetooth's Gateway Smart Starter Kit ${ }^{36}$ provides guidance on how to connect Bluetooth ${ }^{\circledR}$ sensors to IoT via the web using Bluetooth RESTful APIs by creating a simple Bluetooth gateway on a Raspberry PI board. This kit also shows how to move data from all of Bluetooth sensors into the cloud without a mobile device while giving users the ability to communicate and control all of them from one place in the web.

\section{IoT and Security Management}

Growth in loTs brings increased security risks. According to Symantec ${ }^{37}$, loT systems are often highly complex, requiring end-toend security solutions that span cloud and connectivity layers. Resource-constrained loT devices are not sufficiently powerful to support traditional security solutions. Symantec suggests that comprehensive loT security should encompass four cornerstones: (i) communications protection - requires encryption and authentication for devices (e.g. elliptic curve cryptography in resource constrained chips like 8 bit, $8 \mathrm{MHz}$ chips of loT, and embedded "device certificate" keys into loT devices (e.g. embedment of X.509 certificates $^{38}$ into a hardware device during manufacturing, which allows service providers to perform strong authentication to prevent unauthorized devices from obtaining access to services), helping mutually authenticate a wide range of devices including cellular base stations, televisions, and etc...; (ii) devices protection - requires code signing, ensure all code is authorised to run and runtime protection. Code signing cryptographically ensures that codes are not overwritten and host protection in order to protect devices after code has begun running. This entails the verification that all codes running on each loT device have been authorised for that device, and protected by a strong Root of Trust; (iii) devices management - over-the-air (OTA) manageability must be built into the devices before they are shipped; (iv) understanding of system - it is imperative to have an loT Security Analytics capability to understand one's network by flagging anomalies that might be suspicious, dangerous or malicious.

\section{UNDERLYING CONCEPT AND METHODOLOGY}

\section{A. Overall Underpinning Concept}

The SMART-ITEM project will be anchored on an adaptation of biomimicry basic life's principles ${ }^{39}$ (see Figure 2 ).

\section{Adapted Biomimicry Life's Principles}

Biomimicry is an emerging approach to innovation that seeks sustainable solutions to human challenges by emulating nature's time-tested patterns and strategies and its goal is to create sustainable products, or processes ${ }^{40}$. The ultimate goal for SMARTITEM is to create conducive conditions (based on biomimicry life's principles depicted in Figure 2) for the development and deployment of an loT-enabled ecosystem system and services which is sustainable, efficient, effective and beneficial to target users and the elderly population. The principles are as follows: (i) Evolve to survive: replicate strategies that work (note: SMARTITEM will be built on Vitrucare and Wellness Layers's success stories on smart health care and also other work listed in Scope 4: Exploitation of previous work; (ii) Be resource efficient: use multi-functional design (as depicted in Figure 1, SMART-ITEM will afford multi-functionalities to support users in their self-empowered independent journey; (iii) User-centred design will be employed throughout the entire lifecycle of SMART-ITEM IoT ecosystem and services; (iv) Be locally attuned and responsive: use feedback loops and leverage cyclic processes cultivate cooperative relationships (amongst the users, and stakeholders in SMARTITEM), use readily available materials (e.g. use and extend Vitrucare and Wellness Layer's existing platform); (v) Adapt to changing conditions: incorporate diversity (e.g. diversity of supporting activities shown in Figure 2), embody resilience through variation redundancy and decentralisation (note: users will be responsible for their own healthy ageing process), maintain integrity through self-renewal (note: this is made possible via continuous improvement and updates); (vi) Integrate development with growth:

${ }^{33}$ https://github.com/nfarina/homebridge

${ }^{34}$ http://www.automatedhome.co.uk/apple/smartthings-raspberry-pi-homebridge-siri-homekit-controlled-smart-home.html

${ }^{35} \mathrm{http}: / /$ razberry.z-wave.me/index.php?id=1

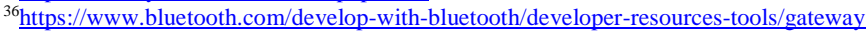

${ }^{37}$ https://www.symantec.com/content/en/us/enterprise/white_papers/iot-security-reference-architecture-wp-en.pdf

${ }^{38} \mathrm{https} / / / \mathrm{www}$. symantec.com/en/uk/device-certificate-service/

${ }^{39} \mathrm{http}: / /$ www.being-here.net/page/2531/applying-biomimicry-to-global-issues

${ }^{40}$ http://biomimicry.org/what-is-biomimicry/ 
build from the bottom-up (note: the SMART-ITEM loT-enabled ecosystem and services will be built based on collated user requirements and analysis), combine modular and nested components (see Figure 1 for SMART-ITEM modular cross-layered architecture).

\section{SMART-ITEM System Architecture Requirements}

The technical requirements for SMART-ITEM layered architecture (shown in Figure 1) will be based on relevant ITU and IOT-A recommended requirements. The Recommendation ITU-T Y.2068 ${ }^{41}$ (2015) has 2068 possible loT requirements which have been coded into the following categories with examples provided by ITU (ibid) and IOT-A ${ }^{42}$ :

\section{(i) Functional Requirements}

Implementation, operability, and integration requirements - interoperability of technologies, applications, services, and management functionalities; autonomous collaboration between devices and services; integration with existing systems using process modelling; involves process execution engine; cross-layered architecture; self-configuration, self-optimisation, selfprotection, self-management and auto-bootstrap management;

Application support requirements - support interworking between different application protocols. OGC (Open Geospatial Consortium) has developed the open SensorThings API ${ }^{43}$ standard to provide an open and unified way to interconnect loT devices, data, and applications over the Web. It builds on Web protocols and the OGC Sensor Web Enablement standards, and applies an easy-to-use REST-like style;

Service requirements - service description (e.g. ITU-T F.744 ${ }^{44}$ - service description and requirements for ubiquitous sensor network middleware), organisation, composition, programmability, orchestration including orchestration engine, meter service reputation, service prioritisation, usage tracking, service subscription according to application domain and implementation;

Communication requirements - autonomous communication, distributed communications, multi-cast messages, communication stacks with small data footprints, low power and lossy network communication, stateless communication methods, commonaddressing-schemes such as IPv6, routing over heterogeneous network, network handover and handoff support, single, simple and standardised management interfaces for all communication protocols, support for intermittent and command-based communication with devices;

Device management requirements - unique identification of devices, resource control, support for sleepy devices, support for multi-homing devices, translational functionality for loT gateways, sensors will be web accessible;

Data management requirements - data acquisition, data filtering, handling, aggregation/fusion, data integrity, data flow modelling, information retrieval, CRUD functions, data freshness;

\section{(ii) non-functional requirements}

Privacy, trust and security requirements - anonymity support, security of data transfer and communications, access control, authentication, user authorisation, encryption, non-repudiation, device security, privacy at atomic level, location privacy, infrastructure services (e.g. resolution services, security services, management services) are trustable, untraceable digital activities, unlinkability, communication confidentiality, communication integrity, pseudonymisation mechanisms, unobservability, data portability, security management - e.g. secure Over-the-Air/Over-the-Network Device Management),

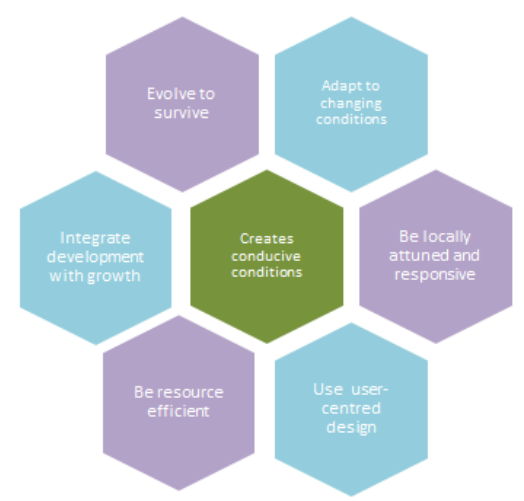

Figure 2: Adaptation of Biomimicry basic life's principles ${ }^{1}$ for SMART-ITEM identity management (e.g. identity and access control - subject-based, role-based or attribute-based; multi-party authentication and cryptography in the IoT; group authentication and authorisation; authorisation, authentication and credentials requirements; uniquely addressable; ownership transfer; anonymous devices and blinded identities;

Semantics requirements - semantic description of physical entities and services, self-description of things (e.g. sensors, actuators, and processors) using standard encoding or model language (e.g. OGC's Encoding Standard, SensorML ${ }^{45}$ );

Resilience requirements - performance; reliability - support the appropriate level of reliability in aspects such as communication, service and data management capabilities to meet system requirements; fault

${ }^{41}$ http://www.itu.int/rec/T-REC-Y.2068-201503-I

${ }^{42}$ http://www.iot-a.eu/public/requirements/copy_of requirements

${ }^{43}$ http://ogc-iot.github.io/ogc-iot-api/

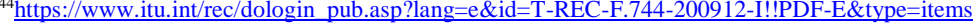

${ }^{45}$ http://www.opengeospatial.org/standards/sensorml 
tolerance; availability; integrity; scalability; evolvability; timeliness; modularity - support components that can be combined in different configurations to form systems as needed;

Extensibility requirements - use of external computing resources, for example, the cloud, for information processing;

Quality of Service (QoS) requirements - transport (aka network) layer fairness);

Accessibility and usability requirements - support end user accessibility/usability preferences and requirements based on UserCentred Design and accessibility/usability based on: W3C guidelines ${ }^{46}$ and IEC/PAS 62883:2014(E) ${ }^{47}$ which specifies a framework for adaptive handling of explicit interaction among humans and AAL spaces. This will also include usability for developers of IoT systems who will be involved in the configuration, operation, and management of the system;

Discoverability requirements - support discovery services for sensors and sensor data;

Awareness requirements - location awareness: e.g. location and distance; locating physical entities based on geographical parameters, standardised location model, location information representation; time-awareness - should support time synchronicity among the actions of interconnected components when using communication and service capabilities; context awareness: enable flexible, user customised and autonomic services based on the related context of loT components and/or users; content awareness: to facilitate services such as path selection and routing of communications based on content);

Manageability - support management capabilities to address aspects such as data management, device management, network management, risk management (e.g. end point controls, gateway controls, network controls, cloud and application controls, overall systems controls), and interface maintenance and alerts;

Energy awareness requirements - manage energy consumption of devices, applications, networks, limit communication range, limit local processing and storage capacity (note: this is the reason for moving these functionalities to the cloud), support sleeping modes;

Standards, and Compliances Requirements - regulation compliances, standard compliance, etc... OGC Standards in the Sensor Web Enablement (SWE) ${ }^{48}$ framework are as follows: Observations \& Measurements (O\&M) - general models and XML encodings for observations and measurements; PUCK Protocol Standard - defines a protocol to retrieve a SensorML description, sensor "driver" code, and other information from the device itself, thus enabling automatic sensor installation, configuration and operation; Sensor Model Language (SensorML) - standard models and XML schema for describing the processes within sensor and observation processing systems; Sensor Observation Service (SOS) - open interface for a web service to obtain observations and sensor and platform descriptions from one or more sensors; Sensor Planning Service (SPS) - an open interface for a web service by which a client can determine the feasibility of collecting data from one or more sensors or models and submit collection requests; SWE Common Data Model - defines low-level data models for exchanging sensor related data between nodes of the OGC $^{\circledR}$ Sensor Web Enablement (SWE) framework; SWE Service Model -- defines data types for common use across OGC Sensor Web Enablement (SWE) services. oneM2M ${ }^{49}$ develops standards for $\mathrm{M} 2 \mathrm{M}$ and IoTs and published technical specifications which address the need for a common M2M Service Layer - functional architecture, technical requirements, security solutions, service layer core protocol specification, management enablement, CoAP protocol binding, HTTP protocol binding, MWTT protocol binding, common terminology, interoperability testing. ISO/IEC 29182 encompasses Sensor Network Reference Architecture (SNRA) for sensor networks and it consists of 7 parts namely: ISO/IEC 29182-1: 2013 ${ }^{50}$ (General Overview and Requirements for sensor networks); ISO/IEC 29182-2: $2013^{51}$ (Vocabulary and Terminology); ISO/IEC 29182-352 (Reference Architecture Views); ISO/IEC 29182-4:2013 ${ }^{53}$ (Entity Models); ISO/IEC 29182-5:2013 ${ }^{54}$ (Interface Definitions); ISO/IEC 29182-655 (Applications); ISO/IEC 29182-7 $7^{56}$ (Interoperability Guidelines). IEEE has published standards for loT $^{57}$ and some of examples of relevant standards are for: Ethernet, Telecommunications and Information Exchange Between Systems, Air Interface for Broadband Wireless Access Systems, Scalable Storage Interface, Smart Transducer Interface for Sensors and Actuators, Interconnecting Distributed Resources with Electric Power Systems, Monitoring, Information Exchange, and Control of Distributed Resources Interconnected with Electric Power Systems, Design, Operation, and Integration of Distributed Resource Island Systems with Electric Power Systems, etc...;

Impact requirements - support components, services, and capabilities which lead to minimal environmental impact but optimal economic as well as social impact of an implementation.

${ }^{46} \mathrm{https}: / /$ www.w3.org/WAI/intro/usable

${ }^{47}$ https://webstore.iec.ch/webstore/webstore.nsf/publication/7577

${ }^{48} \mathrm{http}: / /$ www.opengeospatial.org/domain/swe\#standards

${ }^{49} \mathrm{http}: / /$ www.onem $2 \mathrm{~m}$.org/about-onem $2 \mathrm{~m} /$ why-onem $2 \mathrm{~m}$

${ }^{50} \mathrm{https}: / /$ webstore.iec.ch/preview/info_isoiec29182-1\%7Bed1.0\%7Den.pdf

${ }^{51}$ https://webstore.iec.ch/preview/info_isoiec29182-2\%7Bed1.0\%7Den.pdf

${ }^{52}$ https://webstore.iec.ch/preview/info_isoiec29182-3\%7Bed1.0\%7Den.pdf

${ }^{53} \mathrm{https}: / /$ webstore.iec.ch/preview/info isoiec29182-4\%7Bed1.0\%7Den.pdf

${ }^{54}$ https://webstore.iec.ch/preview/info_isoiec29182-5\%7Bed1.0\%7Den.pdf

${ }_{55}^{5}$ https://webstore.iec.ch/preview/info_isoiec29182-6\%7Bed1.0\%7Den.pdf

${ }^{56} \mathrm{https}: / /$ webstore.iec.ch/preview/info_isoiec29182-7\%7Bed1.0\%7Den.pdf

${ }^{57}$ http://standards.iee.org/innovate/iot/stds.html 


\section{SMART-ITEM System Architecture}

The high level SMART-ITEM System Layered Architecture with functional entities is shown in Figure 1. physical entities in a sensor network consist of hardware, actual devices, sensor nodes, gateways, components, etc... while functional entities represent tasks that may be carried out by or on the physical entities ${ }^{58}$.

SMART-ITEM detailed Architecture (in Figure 3) is adapted from the sensor network functional architecture in ISO/IEC 29182-359.
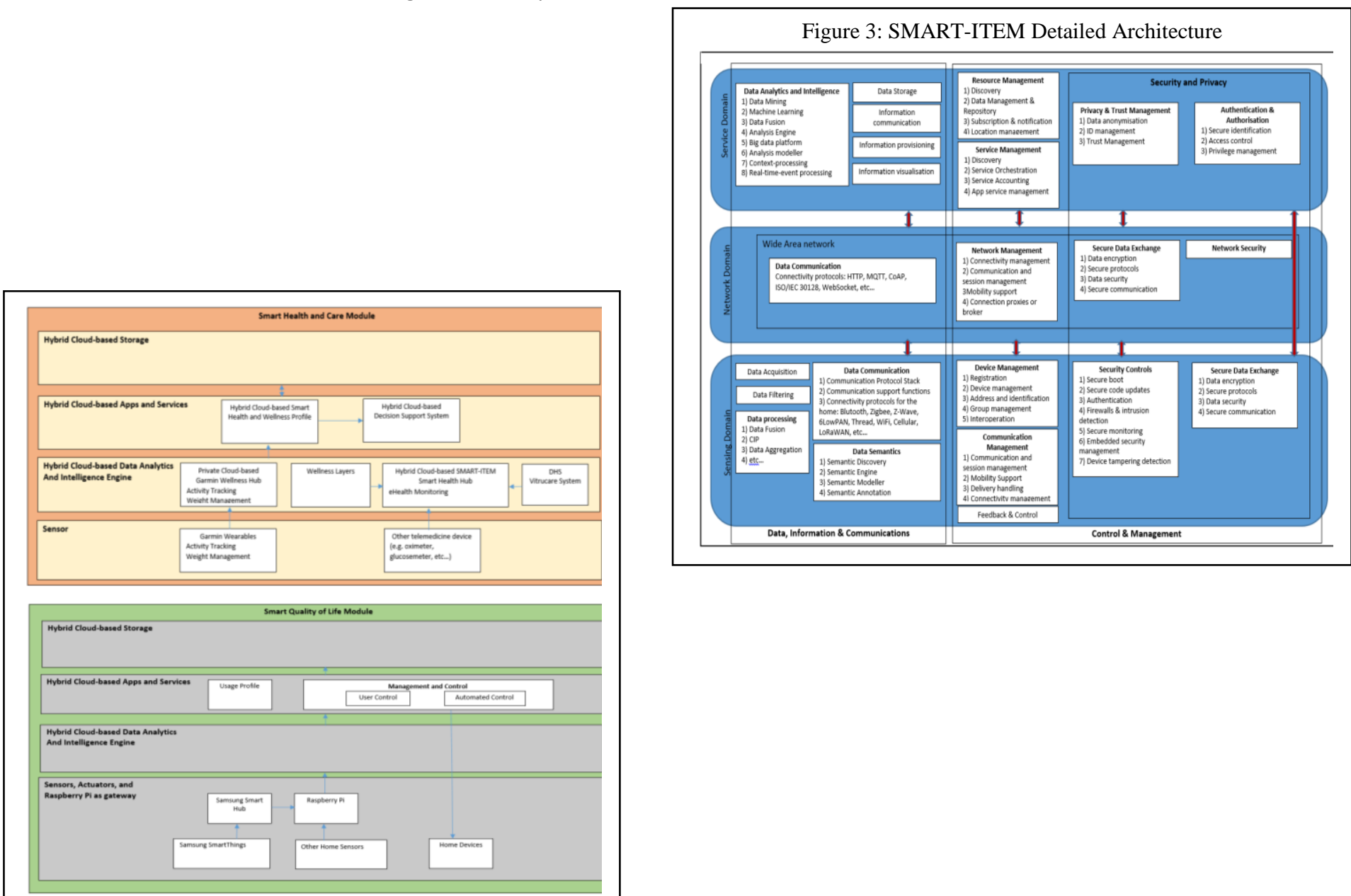

Figure 4. Data Flow in the Smart health \& care and Smart Living modules

The three domains in the functional architecture are: sensing domain, network domain and service domain. The core functions of the system are coded into two broad categories:

\section{Data, Information, and Communications-related functions:}

(i) Data acquisition (or collection) from the sensors, Data filtering based on selected criteria; (iii) Data processing - data aggregation (i.e. raw data collected from a range of sensors); data fusion (i.e. combining and process aggregated data); (iv) Data analytics and intelligence (i.e. to extract feature from the data using data mining techniques, supervised or unsupervised machine learning algorithms, etc...) which results in information or intelligence that will trigger off an appropriate event and could be visualised in various modes such as pie charts, graphs, etc...; (v) Data storage; (vi) Data communication - this involves the transmission of data (e.g. from the sensing layer to the data layer, from the data layer to the platform layer, etc...) and this encompasses connectivity protocols for home devices and also for the network; (vii) Data Semantics - encompasses description and meaning of data;

\section{Control and Management-related functions:}

(i) Resource Management - provides efficient and effective management of loT resources (e.g. discovery of devices and services, location awareness, etc...); (ii) Service Management - supports service orchestration, discovery, app service management to support users, etc...; (iii) Device Management - encompasses functions such as: registration, device configuration, address and

${ }^{58} \mathrm{https}: / /$ webstore.iec.ch/preview/info_isoiec29182-4\%7Bed1.0\%7Den.pdf

${ }^{59} \mathrm{http}: / /$ www.sis.se/PageFiles/15118/Study\%20Report\%20on\%20IoT\%20Reference\%20Architectures\%20and\%20Frameworks.pdf 
identification, management of a group of devices; (iv) Security and Privacy - some of the relevant functions are: privacy and trust management, authentication and authorisation, secure data exchange using encryption and secured protocols, security controls through firewalls, intrusion detection, runtime verification and malware detection, key management, device tampering detection, and boostrapping.

Figure 4 depicts the data flow for the Smart Health and Care as well as the Smart Living modules. The first image shows that integration of Garmin Wellness Portal, Wellness Layers Platform, DHS Vitrucare Platform and SMART-ITEM platform. On the other hand, the scond image shows the integration of Samsung SmartThings sensor data and other home automation sensor data (e.g. air quality, temperature, etc..) using a Raspberry PI 3+ integrated gateway which will be customised for the project so that it will be interoperable for a range of communication protocols (e.g. ZigBee, Bluetooth, Z-Wave, etc...). Data processing, analytics and storage will be executed in private clouds across 8 cities (note: these are hosted in academic/research institutions by coshared with city councils or medical organisations) in the first instance to save costs but will be later migrated to the Amazon EC2 for scalabilityand sustainability purposes.

\section{SMART-ITEM Open Platform}

The SMART-ITEM open platform will be developed using security, compliances, and QoS-related tools from existing open platforms (i.e. FIRE+ and FIWARE ${ }^{60}$ ) that are currently exploited by CTI and LTU for their smart cities projects. It will also adopt exisiting standards (e.g. one M2M, ITU/ISO/IEC standards for loT). Examples of loT services enablement ${ }^{61}$ which will be relevant for this project are: (i) Generic Enablers for: Advanced middleware and interfaces to Network and Devices; Advanced Web-based User Interface; Applications/Services and Data Delivery; Cloud Hosting; Data/Context Management; Security; (ii) Backend Device Management GE ${ }^{62}$; IoT Data Edge Consolidation ${ }^{63}$ (IDEC) GE - addresses the need to process data in real time. Implemented features include filtering, aggregating and merging real-time data from different sources. It is fully integrated with other enablers of FIWARE, especially using the Open Mobile Alliance (OMA) Next Generation Service Interface Context Enabler (NGSI 9 / NGSI 10) which is a very useful and easy format to encapsulate all data and events from RFID tags, Zigbee or IETF devices, as many other smart things.

UniversAAL ${ }^{64}$ aims to create an open platform and standards necessary for the development of Ambient Assisted Living solutions ${ }^{65}$. Security for the different layers in a universAAL platform (which are relevant to SMART-ITEM system and services) are: platform services security - basic security such as user authentication, authorization, secure communication, and functional manifests; application services security - provide value added security to the universAAL platform such as document encryption and consent management; container security - container-specific security features (e.g. provided by OSGi or Android) where OSGi security contributes to the security of the universAAL infrastructure such as OSGi service bundle signing, bundle permission management, and sandboxing. OSGi security also supports some of the security platform services such as secure communication; Java security - provides the Java security model such as the Java sandbox and basic security primitives (note: this is further enhanced with third party cryptographic libraries); device and operating system security which provides a secure environment for computing and data storage by ensuring a trustworthy execution environment, separation of applications and user accounts, etc. UniversAAL and its tools ${ }^{66}$ could be exploited to create, run and publish universAAL applications.

\section{B. Methodology}

The SMART-ITEM project will employ the following integrated approach/methodology: (i) user-centred design approach; (ii) agile methodology for software development (integrated with PDSA (plan, do, study, act) with 3 iterative cycles with 3 years); (iii) integrated software architecture.

\section{User-Centred Design Approach}

According to Norman, et. al. [1], there are two sides to a system interface namely: system perspective and user perspective. The system perspective is changed through proper design while the user perspective is changed through training and experience. User experience will be positive if the user has a good conceptual understanding of the system which has been designed and built around the user needs and requirements (user requirement analysis). Thus, the user-centred design which focuses on the user's needs will entail the following [2]: user requirements analysis, activity/task analysis (necessary for the user interaction); initial testing and evaluation, iterative designs.

\section{Integrated Agile Methodology for Software Development (with PDSA)}

\footnotetext{
${ }^{60} \mathrm{https}$ ://www.fiware.org/2015/03/25/fiware-a-standard-open-platform-for-smart-cities/

${ }^{61}$ http://catalogue.fiware.org/chapter/internet-things-services-enablement

${ }^{62} \mathrm{http}: / /$ catalogue.fiware.org/enablers/backend-device-management-idas

${ }^{63}$ http://catalogue.fiware.org/enablers/iot-data-edge-consolidation-ge-cepheus

${ }^{64} \mathrm{http} / / /$ ercim-news.ercim.eu/en87/special/universaal-an-open-platform-and-reference-specification-for-building-aal-systems

${ }^{65}$ https://github.com/universAAL/platform/wiki

${ }^{66}$ https://github.com/universAAL/platform/wiki/RD-Available-Tools
} 


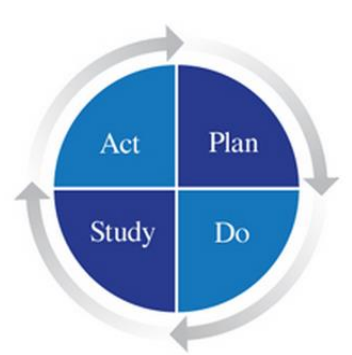

Figure 5: PDSA Cycle

The PDSA Cycle (also known as Deming Wheel/Cycle) ${ }^{67}$ is a systematic series of steps for continuous improvement of the SMART-ITEM IoT-enabled ecosystem and services. The steps are: (i) Plan: identify a goal or purpose, define success metrics, and action plan; (ii) Do: implement action plan (e.g. make a product); (iii) Study: monitor outcomes, validate the plan, identify areas for improvement; (iv) Act: revise step (i) based on results in step (iii). These four steps are iterated as part of a continuous improvement cycle.

Agile methodology [3][5] for software development will be adopted for this project due to its simplicity, flexibility, and adaptability. Just like PDSA, the agile methodology is iterative and incremental, with every aspect of the development lifecycle (i.e. requirements, design, implementation, evaluation, etc...) being continually revisited for continuous improvement. In the agile paradigm, it will provide greater user satisfaction because it delivers a potentially consumable solution for each iteration and enables users to enhance (i.e. evolve for the better) their requirements throughout the entire project [3]. Additionally, it has a quicker delivery compared with the traditional waterfall methodology. Figure 6 depicts our integrated agile methodology and PDSA. The steps involved are: (i) PLAN: Define requirements (Research) - user requirements: elderly population (greater or equal

Figure 6: Integrated Agile Lifecycle and PDSA (extracted from [6])

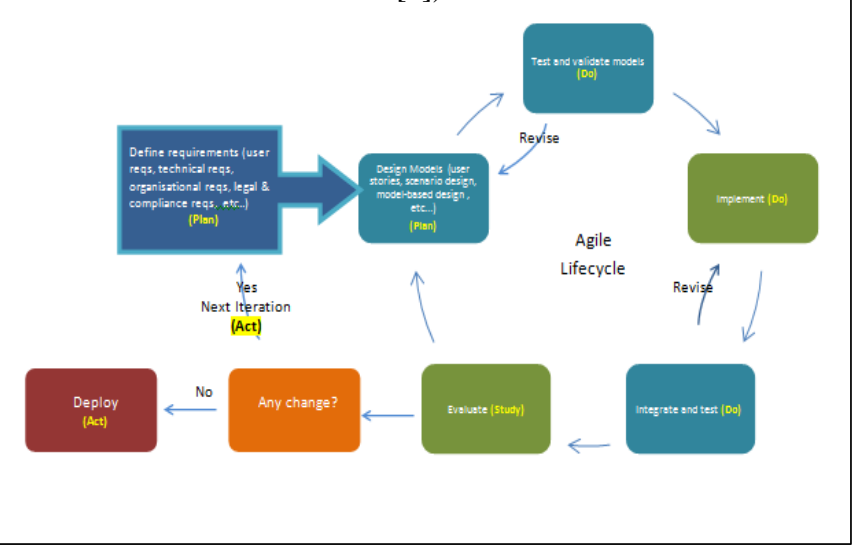

65 years old) sample from Leeds City Council, Phoenix Medical Practice, Municipality of Patras, Municipality of Zakynthos, Nancy City (via Ecogelec), Lulea City (via EIP), Municipality of Zilina, Kiev City Council; technical requirements; standards; legal and compliance requirements (for all the participating countries); (ii) PLAN: Design models (Design) - user stories (i.e. begins with understanding the user, capture it in the form of a persona (a tool to help visualise the user and needs, followed by using the tasks and goals of the "personas" to support decisions on system functionalities ${ }^{68}$ ), scenario-based designs (i.e. grounding design in an understanding and capturing the interrelationships of tasks users carry out over time) ${ }^{69}$, model-based designs (e.g. Object, View, and Interaction Design, or OVID [4] which systematically builds models using based on a specification of the user model (Design, Modelling, and Architecture); (iii) DO: Test and verify design models (Design, Modelling and Architecture) in (ii) using simulations or experimental environment; (iv) DO: Implement (Development and Integration) - Develop system and services for the real environment with at least 3 complete cycles (shown in Figure 7) for SMART-ITEM web portal, modules ( SMART health and care (with secured individual personalised view), Smart Quality of Life, and SMART-ITEM Community of Support); (v) DO: Integrate and Test (Development and Integration) - integration within and between the 6 layers: Sensing Layer, Data Layer, Platform Layer (i.e. Data Access Layer and Cloud Integration Layer), Application and Services Layer, Secured Network Layer, and Access Layer); (vi) Study: Evaluation of SMART-ITEM System and Services (Evaluation: Research) - expert appraisal, users, impact analyses (Evaluation: Impact Analyses); (vii) Act: Next iteration if revision is required (note: the entire cycle will be repeated -

\footnotetext{
${ }^{67}$ https://www.deming.org/theman/theories/pdsacycle

${ }^{68} \mathrm{http}: / /$ usability-bremen.de/wp-content/uploads/2013/03/Winter-Holt-et-al-2012-Persona-driven-agile-development.pdf

${ }^{69}$ http://www.ie.zjut.edu.cn/htm/uploadfiles/2007101052010670.pdf
} 
possible evolution of requirements, revision of designs, changes in implementation, etc...). On the other hand, if the evaluation results meet the SMART-ITEM system and services goals, then, it shall be deployed in the real environment.

\section{SMART-ITEM Integrated Software Architecture}

SMART-ITEM integrated software architecture (depicted in Figure 1) is modular and cross-layered. The design and development of each module in the SMART-ITEM system and services will be based on the component-based software design and development approach ${ }^{70}$ with the following principles: (i) components are independent (note all the SMART-ITEM modules can be developed independently at the initial stage; (ii) communication is through well-defined interfaces (note: in SMART-ITEM, the interaction amongst the modules are made possible via integrations; (iii) components have shared platforms (note: in SMART-ITEM, a cloudbased platform will be used). As previously discussed, the architecture consists of 6 layers (i.e. Sensing Layer, Data Layer, Platform Layer, Application and Services Layer, Secured Network Layer, and Access Layer) and 3 modules (Smart Health and Care, Smart Quality of Life, SMART-ITEM Community of Support). The Microsoft .NET framework will be employed to support the access and application/services layers because it will support the software required for the development of all the modules. Additionally, this will facilitate deep customisation, scalable, and integration. The flexible structures will be cost-effective and allow timely deployment (as in the agile methodology). Note that the same consortium partners will be involved throughout the lifecycle of a particular module they are responsible for.

\section{Pilot Study for the Design, Development, and Deployment of SMART-ITEM System and Services}

Users will be employed for the pilot tests (i.e. age must be more than 65 years of age living at home or in a care home). Initially, a small scale pilot of 50 users will be conducted only within a private cloud environment. Three iterations of large scale pilots are conducted (note: number of target users is 2200). During the first two iterative cycles, only the distributed private clouds (one for each city and hosted in the academic/research institutes) are used. However, during the third iterative cycle, the services are migrated to the public cloud for future scalability and sustainability.

\section{Some Relevant Technologies for SMART-ITEM}

Each SMART-ITEM system element is depicted in Figure 1. Relevant technologies for SMART-ITEM ecosystem system and services are tabulated in Table 1. The system elements are interdependent. The four types of interactions are: data flow; information (processed data) flow; access; integration (between and within layers).

Table 1: Some Key Technologies for SMART-ITEM Layers and Modules

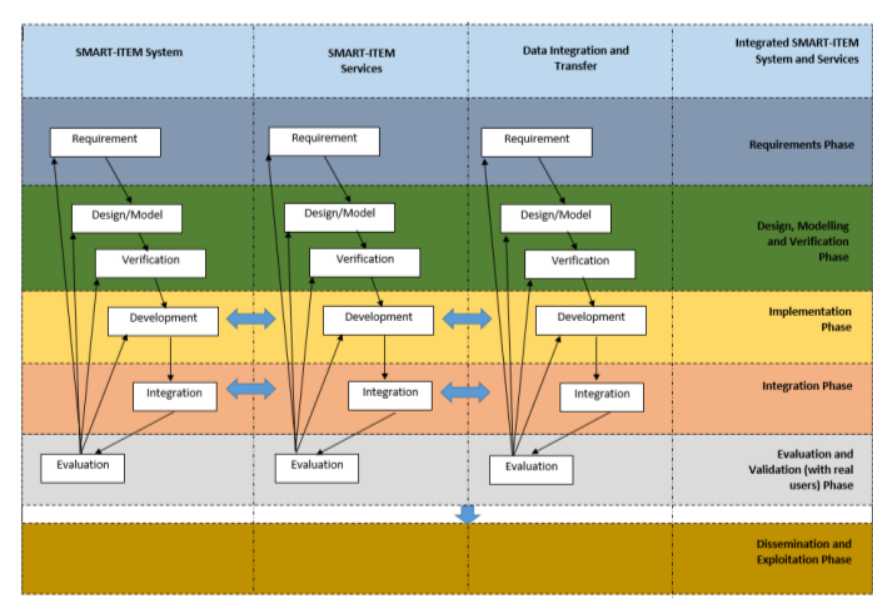

Figure 7: Iterative Component-Based Software Development Lifecycle

\begin{tabular}{|c|c|c|}
\hline Layer/Others & Description & Key Technology \\
\hline Sensing Layer & $\begin{array}{l}\text { Smart Health and Care } \\
\text { Telemonitoring devices } \\
\text { for activity tracking and } \\
\text { vital signs (with wireless } \\
\text { capabilities) }\end{array}$ & $\begin{array}{l}\text { Garmin Vivo smart (activity and } \\
\text { heart rate) vívosmart }{ }^{\circledR} \text { HR (Part } \\
\text { Number: } 010-01955-06 \text { ) } \\
\text { Weighing scale (Garmin Index } \\
\text { Smart Scale - White) }\end{array}$ \\
\hline
\end{tabular}

\footnotetext{
${ }^{70}$ http://cs.ecs.baylor.edu/ maurer/CSI5v93CLP/Papers/JAsCo.pdf
} 


\begin{tabular}{|c|c|c|}
\hline & & $\begin{array}{l}\text { CONTEC CMS50EW Color OLED } \\
\text { Bluetooth Fingertip SPO2 } \\
\text { Monitoring Pulse Oximeter }\end{array}$ \\
\hline & $\begin{array}{l}\text { Smart Quality of Life } \\
\text { Smart home automation }\end{array}$ & $\begin{array}{l}\text { Raspberry Pi 3: Model B+ } \\
\text { (internet gateway) } \\
\text { SAMSUNG SmartThings Starter } \\
\text { Kit } \\
\text { Samsung Galaxy Grand Prime } \\
\text { DUAL SIM Simfree } 5 \text { Inch } \\
\text { Touchscreen Smartphone - Gold }\end{array}$ \\
\hline Data Layer & $\begin{array}{l}\text { Data integration (for the } \\
\text { telemonitored data and } \\
\text { patient health record } \\
\text { data) and data storage }\end{array}$ & $\begin{array}{l}\text {.NET Framework for data } \\
\text { integration of XML and } \\
\text { relational data using ADO.NET. } \\
\text { A list of data integration } \\
\text { solutions in the cloud: } \\
\text { proprietary solutions (e.g. } \\
\text { Informatica, Pendaho, } \\
\text { SnapLogic, Azure Biztalk } \\
\text { Services, etc...); open sourced } \\
\text { technologies for data } \\
\text { integration (e.g. JSON, XML, } \\
\text { Hadoop and Apache Spark, } \\
\text { etc...). Open-source web/cloud- } \\
\text { based MySQL will be used for } \\
\text { data storage. }\end{array}$ \\
\hline Platform Layer & Platform & $\begin{array}{l}\text { Cloud-based platform - } \\
\text { Microsoft Azure which supports } \\
\text { the Microsoft .NET framework. }\end{array}$ \\
\hline \multirow[t]{5}{*}{$\begin{array}{l}\text { Application and } \\
\text { Services Layer }\end{array}$} & $\begin{array}{l}\text { Database, access, and } \\
\text { manipulation }\end{array}$ & $\begin{array}{l}\text { PHP and MYSQL in Azure, XML } \\
\text { and C\# (for simple and scalable } \\
\text { data manipulation); }\end{array}$ \\
\hline & $\begin{array}{l}\text { Data Analytics and } \\
\text { Visualisation }\end{array}$ & $\begin{array}{l}\text { Python for .NET (pythonnet), } \\
\text { IronPython, R.NET (statistical } \\
\text { language). }\end{array}$ \\
\hline & Expert System & $\begin{array}{l}\text { CLIPS rule-based programming } \\
\text { language, CLIPS.NET (user } \\
\text { interface for CLIPS), fuzzyclips. }\end{array}$ \\
\hline & Machine Learning & $\begin{array}{l}\text { Infer.NET (bayesian network), } \\
\text { Accord.NET framework, } \\
\text { Machine Learning Python } \\
\text { (MLPY). }\end{array}$ \\
\hline & Social Community & $\begin{array}{l}\text { Social Networking in .NET, } \\
\text { ASP.NET Discussion Forum, } \\
\text { ASP.NET Community Suite. }\end{array}$ \\
\hline Access Layer & User Interface & $\begin{array}{l}\text { Jquery, Ajax (for enhanced } \\
\text { interactivity and } \\
\text { responsiveness), ASP.NET, } \\
\text { C\#.NET, APIs. }\end{array}$ \\
\hline \multirow[t]{2}{*}{$\begin{array}{l}\text { Cloud } \\
\text { infrastructures }\end{array}$} & $\begin{array}{l}\text { Private cloud (servers and } \\
\text { storage) }\end{array}$ & $\begin{array}{l}\text { HP ProLiant DL380p G8 } \\
\text { Storage } \\
\text { HDD Tray Hard Drive Caddy } \\
2.5 " \text { : } \\
\text { Intel DC S3710 Series 200GB } \\
2.5^{\prime \prime} \\
\text { Seagate ST2000NX0273 2.5- } \\
\text { Inch } 2 \text { TB Internal Hard Drive } \\
\text { RAM } \\
\text { 16GB RAM Memory for HP- } \\
\text { Compaq ProLiant DL380p G8 } \\
\text { (DDR3-10600 - Reg): }\end{array}$ \\
\hline & Public Cloud and services & $\begin{array}{l}\text { Amazon EC2 (Europe, Ireland) - } \\
\text { web portal } \\
\text { Amazon S3 (Europe, Ireland) - } \\
\text { storage } \\
\text { Amazon SNS (Europe, Ireland) - } \\
\text { Simple Notification Service } \\
\text { AWS Data Transfer Out } \\
\text { AWS Data Transfer In } \\
\text { AWS IoT Suite }\end{array}$ \\
\hline Modelling & Simulations & Matlab, and Simulink. \\
\hline
\end{tabular}

Impact Analyses

Different categories of loT technology impact assessment have been tabulated in Table 2 .

Table 2 Impact Assessment Methods 


\begin{tabular}{|c|c|}
\hline Economic Impact Assessment & $\begin{array}{l}\text { Benefit/costs Analysis } \\
\text { B/C = Present Value of Project Benefit divided by Present Value of Project Cost By definition } \\
\text { Cost Effectiveness } \\
\text { This is the ratio of cost per unit of desired results (e.g. cost per person use). This test is applicable when the benefit measure cannot } \\
\text { be reliably translated into money terms (e.g. carbon footprint reduction). It is most usefully applied when there is a clear goal } \\
\text { (measure) for the desired level of benefit results. } \\
\text { Net Present Value } \\
\text { NPV = Present Value of Project Benefit minus the Present Value of Project Cost (Present Value is at the point of decision making). } \\
\text { Gross Value Added } \\
\text { GVA - measures the contribution to an economy of an individual producer, industry, sector or region. It is used in the calculation of } \\
\text { gross domestic product (GDP). } \\
\text { Return on Investment } \\
\text { Benefits derived from investment on some resources. }\end{array}$ \\
\hline $\begin{array}{l}\text { Environmental } \\
\text { assessment }\end{array}$ & $\begin{array}{l}\text { ISO } 14001 \text { - identify and evaluate environment assessment of loT solutions. } \\
\text { The lifecycle assessment of the loT technology would be conducted according to ETSI's LCA methodology }{ }^{71} \text { for Environmental } \\
\text { Engineering (EE); Life Cycle Assessment (LCA) of ICT equipment, networks and services. }\end{array}$ \\
\hline $\begin{array}{c}\text { Health informatics impact } \\
\text { assessment }\end{array}$ & Examples are time efficiency, user effectiveness, user behavioural change, etc... \\
\hline
\end{tabular}

\section{CONCLUSION}

The SMART-ITEM project will be a valuable project because of the scale and variety of potential users. The pilot target audience for SMART-ITEM use cases across 8 cities in 6 countries is 2200 users. Secondly, users are co-creators of health and wellbeing. The project involves the development of an innovative and seamless integrated loT-enabled ecosystem and services to support smart living. The The SMART-ITEM system and services complex modular and vertically cross-layered architecture consists of 3 modules and 6 layers (see Figure 1). Currently, integrating loT solutions from different providers are challenging because they are accompanied with proprietary apps and web access. Additionally, the interoperability between the different loT devices poses another challenge due to the varying communication protocols. Using a customised Raspberry PI 3+ as an integrated gateway for different sensors data will be beyond the state-of-the-art.

\section{REFERENCES}

[1] Norman, D.A., et. al. (1986). User Centered System Design: new Perspectives on Human-Computer Interaction, Hillsdale: Lawrence Erlbaum Associates Publishers.

[2] Ritter, F. E., et al. (2014). Foundations for Designing User-Centered Systems, Springer-Verlag London, DOI: 10.1007/978-1-4471-5134-0_2.

[3] IBM. (2012). Agile For Dummies ${ }^{\circledR}$, IBM Limited Edition, Published by John Wiley \& Sons, Inc.

[4] Roberts, D., Berry, D., Isensee, S., Mullaly,J. (1998). Designing for the user with OVID, MacMillan

[5] http://agilemethodology.org/

[6] Kor, A. L., et. al. (2015). An Integrated Healthcare System and Services for Empowering Patients, International Conference on Health Informatics and Medical Systems, July 27-30, 2015, Las Vegas, USA

\footnotetext{
${ }^{71}$ http://www.etsi.org/deliver/etsi_ts/103100_103199/103199/01.01.01_60/ts_103199v010101p.pdf
} 